\title{
Correction to: Expressiveness of component-based frameworks: a study of the expressiveness of BIP
}

\author{
Eduard Baranov $^{1}$ (D) . Simon Bliudze ${ }^{2}$ (D)
}

Published online: 16 September 2019

(c) Springer-Verlag GmbH Germany, part of Springer Nature 2019

\section{Correction to: Acta Informatica https://doi.org/10.1007/s00236-019-00337-7}

The Original Article has been partially funded by Hauts-de-France regional STaRS programme.

Publisher's Note Springer Nature remains neutral with regard to jurisdictional claims in published maps and institutional affiliations.

The original article can be found online at https://doi.org/10.1007/s00236-019-00337-7.

Simon Bliudze

simon.bliudze@inria.fr

Eduard Baranov

eduard.baranov@uclouvain.be

1 Université catholique de Louvain, Place de l'Université 1, 1348 Ottignies-Louvain-la-Neuve, Belgium

2 INRIA Lille - Nord Europe, 40 avenue Halley, 59650 Villeneuve d'Ascq, France 